\title{
The Discrete Poisson-Amarendra Distribution
}

\section{Rama Shanker}

Department of Statistics, Eritrea Institute of Technology, Asmara, Eritrea

\section{Email address:}

shankerrama2009@gmail.com

\section{To cite this article:}

Rama Shanker. The Discrete Poisson-Amarendra Distribution. International Journal of Statistical Distributions and Applications. Vol. 2, No. 2, 2016, pp. 14-21. doi: 10.11648/j.jjsd.20160202.11

Received: June 4, 2016; Accepted: June 29, 2016; Published: August 26, 2016

\begin{abstract}
In this paper, a Poisson mixture of the Amarendra distribution, introduced by Shanker (2016 c), is proposed, and called the, "Poisson-Amarendra distribution". The first four raw moments (about the origin) and central moments (about the mean) are obtained. The expression for coefficient of variation, skewness and kurtosis are also given. For the estimation of its parameter, the maximum likelihood estimation and the method of moments are discussed. Moreover, the distribution is fitted using maximum likelihood estimate to certain data sets to test its goodness of fit over Poisson, Poisson-Lindley and PoissonSujatha distributions. The corresponding fitting are found to be quite satisfactory in almost all data sets.
\end{abstract}

Keywords: Amarendra Distribution, Sujatha Distribution, Poisson-Lindley Distribution, Poisson-Sujatha Distribution, Compounding, Moments, Estimation of Parameter, Goodness of Fit

\section{Introduction}

The probability density function (p. d. f.) and the cumulative distribution function (c. d. f.) of Amarendra

$$
\begin{gathered}
f(x ; \theta)=\frac{\theta^{4}}{\theta^{3}+\theta^{2}+2 \theta+6}\left(1+x+x^{2}+x^{3}\right) e^{-\theta x}, x>\mathbf{0}, \theta>\mathbf{0} . \\
F(x, \theta)=\mathbf{1}-\left[\mathbf{1}+\frac{\theta^{3} x^{3}+\theta^{2}(\theta+3) x^{2}+\theta\left(\theta^{2}+2 \theta+\mathbf{6}\right) x}{\theta^{3}+\theta^{2}+2 \theta+6}\right] e^{-\theta x}, x>\mathbf{0}, \theta>\mathbf{0} .
\end{gathered}
$$

It has been shown by Shanker (2016 c) that Amarendra distribution is a four component mixture of exponential $(\theta)$ distribution, a gamma $(\mathbf{2}, \theta)$ istribution, a gamma $(\mathbf{3}, \theta)$ distribution and a gamma $(\mathbf{4}, \theta)$ distribution with their mixing proportions

$$
\begin{gathered}
\frac{\theta^{3}}{\theta^{3}+\theta^{2}+2 \theta+6}, \frac{\theta^{2}}{\theta^{3}+\theta^{2}+2 \theta+6}, \frac{2 \theta}{\theta^{3}+\theta^{2}+2 \theta+6}, \text { and } \\
\frac{6}{\theta^{3}+\theta^{2}+2 \theta+6}
\end{gathered}
$$

respectively. Shanker (2016 c) has discussed its various mathematical and statistical properties including its shape for different values of its parameter, moment generating function, moments, skewness, kurtosis, hazard rate function, mean residual life function, stochastic orderings, mean deviations, Bonferroni and Lorenz curves, some amongst others. Further, Shanker (2016 c) has also discussed the estimation of its parameter using maximum likelihood estimation and method of moments along with applications for modeling lifetime data and observed that it gives much closer fit than Akash, Shanker and Sujatha distributions introduced by Shanker (2015 a, 2015 b, 2016 a,), Lindley (1958) and exponential distributions. It would be worth mentioning that Shanker (2015 a, 2015 b, 2016 a) has proposed Akash, Shanker and Sujatha, distributions along with their various mathematical and statistical properties to model lifetime data arising from engineering and biomedical sciences and showed that these distributions provide much 
closer fit than Lindley and exponential distributions.

In this paper, a Poisson mixture of Amarendra distribution, introduced by Shanker (2016 c), has been proposed, and called the "Poisson--Amarendra distribution (PAD)". Its important mathematical and statistical properties including, shape, raw moments, central moments, coefficients of variation, skewness and kurtosis are obtained and discussed. The estimation of PAD's parameter has been also discussed using both maximum likelihood estimation and method of moments. The distribution is then fitted using its maximum likelihood estimate on certain data sets in order to test its goodness of fit over Poisson distribution, Poisson-Lindley distribution (PLD), a Poisson-mixture of Lindley (1958) distribution, introduced by Sankaran (1970) and PoissonSujatha distribution (PSD) of Shanker (2016 b), a Poisson mixture of Sujatha distribution, introduced by Shanker (2016 a).

\section{Poisson-Amarendra Distribution}

Assuming the parameter $\lambda$ of the Poisson distribution to follows Amarendra distribution (1), the Poisson mixture of Amarendra distribution can be obtained as

$$
\begin{gathered}
\mathrm{P}(X=x)=\int_{0}^{\infty} \frac{e^{-\lambda} \lambda^{x}}{x !} \cdot \frac{\theta^{4}}{\theta^{3}+\theta^{2}+2 \theta+6}\left(1+\lambda+\lambda^{2}+\lambda^{3}\right) e^{-\theta \lambda} d \lambda \\
=\frac{\theta^{4}}{\left(\theta^{3}+\theta^{2}+2 \theta+6\right) x !} \int_{0}^{\infty} \lambda^{x}\left(1+\lambda+\lambda^{2}+\lambda^{3}\right) e^{-(\theta+1) \lambda} d \lambda \\
=\frac{\theta^{4}}{\theta^{3}+\theta^{2}+2 \theta+6} \cdot \frac{x^{3}+(\theta+7) x^{2}+\left(\theta^{2}+\mathbf{5} \theta+\mathbf{1 5}\right) x+\left(\theta^{3}+4 \theta^{2}+7 \theta+\mathbf{1 0}\right)}{(\theta+\mathbf{1})^{x+4}} ; x=\mathbf{0}, \mathbf{1}, 2, \ldots, \theta>\mathbf{0}
\end{gathered}
$$
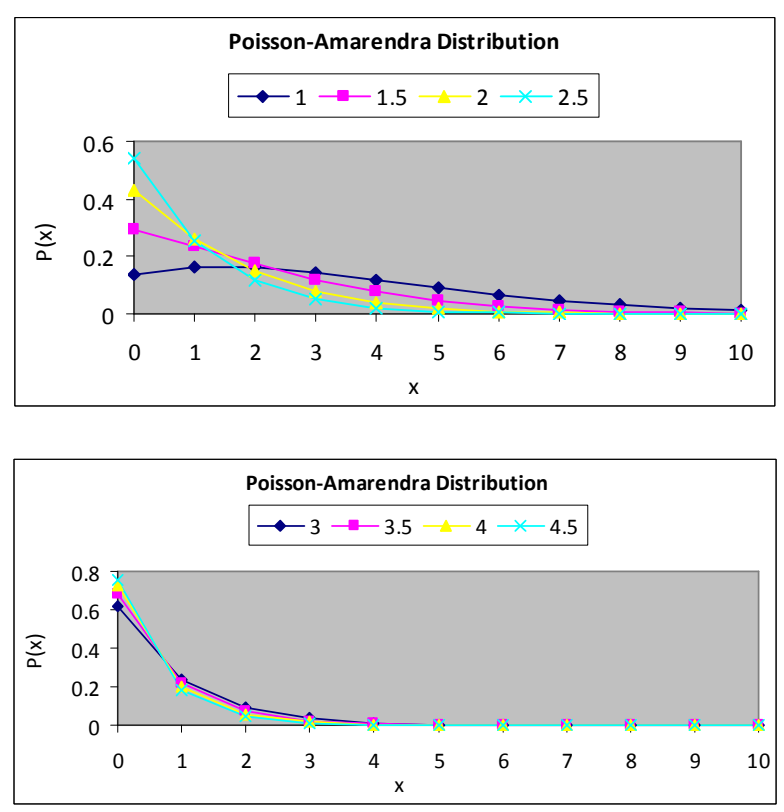

Fig. 1. Graphs of the PAD'p. m. f. for varying values of its parameter $\theta$
We shall refer to this distribution as the "PoissonAmarendra distribution (PAD)". The graphs of the probability mass function (p. m. f) of PAD for varying values of its parameter $\theta$ are shown in the figure 1.

Recall that Sankaran (1970) obtained Poisson-Lindley distribution (PLD) having p. m. f. of the form

$$
P(X=x)=\frac{\theta^{2}(x+\theta+\mathbf{2})}{(\theta+\mathbf{1})^{x+3}} ; x=\mathbf{0}, \mathbf{1}, \mathbf{2}, \ldots, \theta>\mathbf{0}
$$

by compounding Poisson distribution with Lindley distribution, introduced by Lindley (1958) having p. d. f of the form

$$
f(x, \theta)=\frac{\theta^{2}}{\theta+\mathbf{1}}(\mathbf{1}+x) e^{\theta x} ; x>\mathbf{0}, \theta>\mathbf{0} .
$$

Shanker and Hagos (2015) has discussed the applications of PLD for modeling count data-sets from biological sciences and observed that it gives better fit than Poisson distribution.

Shanker (2016 b) obtained Poisson-Sujatha distribution (PSD) with its p. m. f. given by

$$
P(X=x)=\frac{\theta^{3}}{\theta^{2}+\theta+2} \cdot \frac{x^{2}+(\theta+4) x+\left(\theta^{2}+3 \theta+4\right)}{(\theta+1)^{x+3}} ; x=\mathbf{0}, 1,2, \ldots, \theta>\mathbf{0} .
$$

by compounding Poisson distribution with Sujatha distribution, introduced by Shanker (2016 a) having p. d. f. given by

$$
f(x ; \theta)=\frac{\theta^{3}}{\theta^{2}+\theta+2}\left(\mathbf{1}+x+x^{2}\right) e^{-\theta x} ; x>\mathbf{0}, \theta>\mathbf{0}
$$

Sujatha distribution, introduced by Shanker (2016 a) is considered a better model than exponential and Lindley distributions when modeling lifetime data from biomedical science and engineering. Further, Shanker and Hagos (2016 a) provided a detailed study about the application of the Poisson-Sujatha distribution (PSD) when modeling biological science data, and observed a better fit than the Poisson-Lindley distribution (PLD) and Poisson-distribution. 
Shanker and Hagos (2016 b, 2016 c) have obtained the sizebiased Poisson-Sujatha distribution (SBPSD) and zerotruncated Poisson-Sujatha distribution (ZTPSD) and discussed their various mathematical and statistical properties, estimation of their parameter and applications. Further, Shanker and Hagos (2016 d) have also provided a detailed study regarding applications of the zero-truncated Poisson distribution (ZTPD), the zero-truncated PoissonLindley distribution (ZTPLD), and the zero-truncated Poisson-Sujatha distribution (ZTPSD) for modeling data sets (with zero counts excluded) from demography and biological sciences and concluded that in majority of data sets ZTPSD gives better fit than ZTPD and ZTPLD.

\section{Moments and Related Measures}

The $r$ th factorial moment about origin of PAD (4) can be obtained as

$$
\begin{gathered}
\mu_{(r)}{ }^{\prime}=E\left[E\left(X^{(r)} \mid \lambda\right)\right], \text { where } \\
X^{(r)}=X(X-\mathbf{1})(X-\mathbf{2}) \ldots(X-r+\mathbf{1})
\end{gathered}
$$

Using (3) the $r$ th moment about origin of PAD (4) can be obtained as

$$
\begin{gathered}
\mu_{(r)}{ }^{\prime}=E\left[E\left(X^{(r)} \mid \lambda\right)\right] \\
=\frac{\theta^{4}}{\theta^{3}+\theta^{2}+2 \theta+6} \int_{0}^{\infty}\left[\sum_{x=0}^{\infty} x^{(r)} \frac{e^{-\lambda} \lambda^{x}}{x !}\right]\left(1+\lambda+\lambda^{2}+\lambda^{3}\right) e^{-\theta \lambda} d \lambda \\
=\frac{\theta^{4}}{\theta^{3}+\theta^{2}+2 \theta+6} \int_{0}^{\infty}\left[\lambda^{r} \sum_{x=r}^{\infty} \frac{e^{-\lambda} \lambda^{x-r}}{(x-r) !}\right]\left(1+\lambda+\lambda^{2}+\lambda^{3}\right) e^{-\theta \lambda} d \lambda
\end{gathered}
$$

Taking $x+r$ in place of $x$ within bracket, we get

$$
\mu_{(r)}^{\prime}=
$$

$$
\frac{\theta^{4}}{\theta^{3}+\theta^{2}+2 \theta+6} \int_{0}^{\infty} \lambda^{r}\left[\sum_{x=0}^{\infty} \frac{e^{-\lambda} \lambda^{x}}{x !}\right]\left(1+\lambda+\lambda^{2}+\lambda^{3}\right) e^{-\theta \lambda} d \lambda
$$

The expression within the bracket is clearly unity and hence we have

$$
\mu_{(r)}^{\prime}=\frac{\theta^{4}}{\theta^{3}+\theta^{2}+2 \theta+6} \int_{0}^{\infty} \lambda^{r}\left(1+\lambda+\lambda^{2}+\lambda^{3}\right) e^{-\theta \lambda} d \lambda
$$

Using the gamma integral and some algebraic simplification, we get finally a general expression for the $r$ th factorial raw moment (about the origin) of PAD (4) as

$$
\mu_{(r)}^{\prime}=\frac{r !\left[\theta^{3}+(r+1) \theta^{2}+(r+1)(r+2) \theta+(r+1)(r+2)(r+3)\right]}{\theta^{r}\left(\theta^{3}+\theta^{2}+2 \theta+6\right)} ; r=1,2,3, \ldots
$$

Substituting $r=\mathbf{1}, \mathbf{2}, \mathbf{3}$, and $\mathbf{4}$ in (9), the first four raw factorial moments can be obtained and using the relationship between factorial raw moments and the usual raw moments, the first four raw moments the PAD (4) are obtained as

$$
\begin{gathered}
\mu_{1}^{\prime}=\frac{\theta^{3}+2 \theta^{2}+6 \theta+24}{\theta\left(\theta^{3}+\theta^{2}+2 \theta+6\right)} \\
\mu_{2}^{\prime}=\frac{\theta^{4}+4 \theta^{3}+12 \theta^{2}+48 \theta+120}{\theta^{2}\left(\theta^{3}+\theta^{2}+2 \theta+6\right)} \\
\mu_{3}^{\prime}=\frac{\theta^{5}+8 \theta^{4}+30 \theta^{3}+120 \theta^{2}+480 \theta+720}{\theta^{3}\left(\theta^{3}+\theta^{2}+2 \theta+6\right)} \\
\mu_{4}^{\prime}=\frac{\theta^{6}+16 \theta^{5}+84 \theta^{4}+360 \theta^{3}+1680 \theta^{2}+5040 \theta+5040}{\theta^{4}\left(\theta^{3}+\theta^{2}+2 \theta+6\right)}
\end{gathered}
$$

Using the relationship between the central moments (about the mean) and the raw moments (about the origin), the central moments of the PAD (4) are thus given by

$$
\begin{gathered}
\mu_{2}=\frac{\theta^{7}+4 \theta^{6}+14 \theta^{5}+58 \theta^{4}+144 \theta^{3}+156 \theta^{2}+240 \theta+144}{\theta^{2}\left(\theta^{3}+\theta^{2}+2 \theta+6\right)^{2}} \\
\mu_{3}=\frac{\left(\begin{array}{l}
\theta^{11}+7 \theta^{10}+32 \theta^{9}+146 \theta^{8}+582 \theta^{7}+1440 \theta^{6}+2616 \theta^{5}+4416 \theta^{4} \\
+4440 \theta^{3}+4896 \theta^{2}+4320 \theta+1728
\end{array}\right)}{\theta^{3}\left(\theta^{3}+\theta^{2}+2 \theta+6\right)^{3}}
\end{gathered}
$$




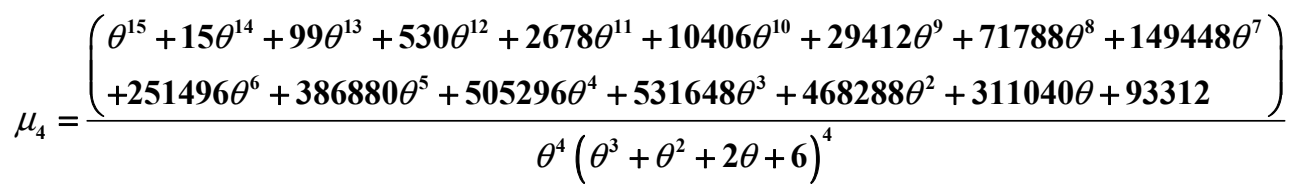

The coefficient of variation $(C . V)$, coefficient of Skewness $\left(\sqrt{\beta_{1}}\right)$, coefficient of Kurtosis $\left(\beta_{2}\right)$ and index of dispersion $(\gamma)$ of the PAD (4) are thus obtained as

$$
\begin{aligned}
& C . V=\frac{\sigma}{\mu_{1}^{\prime}}=\frac{\sqrt{\theta^{7}+4 \theta^{6}+14 \theta^{5}+58 \theta^{4}+144 \theta^{3}+156 \theta^{2}+240 \theta+144}}{\theta^{3}+2 \theta^{2}+6 \theta+24} \\
& \sqrt{\beta_{1}}=\frac{\mu_{3}}{\mu_{2}^{3 / 2}}=\frac{\left(\begin{array}{l}
\theta^{11}+7 \theta^{10}+32 \theta^{9}+146 \theta^{8}+582 \theta^{7}+1440 \theta^{6}+2616 \theta^{5}+4416 \theta^{4} \\
+4440 \theta^{3}+4896 \theta^{2}+4320 \theta+1728
\end{array}\right)}{\left(\theta^{7}+4 \theta^{6}+14 \theta^{5}+58 \theta^{4}+144 \theta^{3}+156 \theta^{2}+240 \theta+144\right)^{3 / 2}}
\end{aligned}
$$

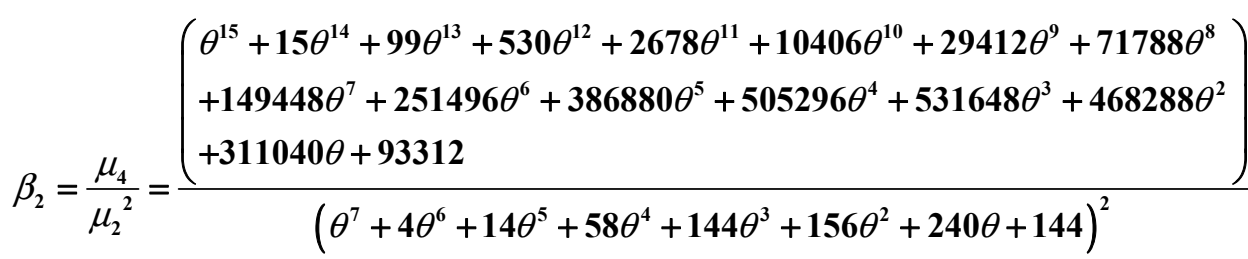

$$
\begin{aligned}
& \gamma=\frac{\sigma^{2}}{\mu_{1}^{\prime}}=\frac{\theta^{7}+4 \theta^{6}+14 \theta^{5}+58 \theta^{4}+144 \theta^{3}+156 \theta^{2}+240 \theta+144}{\theta\left(\theta^{3}+\theta^{2}+2 \theta+6\right)\left(\theta^{3}+2 \theta^{2}+6 \theta+24\right)}
\end{aligned}
$$

To study the nature and behavior of $\mu_{1}^{\prime}, \mu_{2}, \mathrm{C} . \mathrm{V}, \sqrt{\beta_{1}}, \beta_{2}$ and $\gamma$ of the PLD, PSD and PAD, their numerical values are calculated for varying values of the parameter $\theta$ and the results are presented in tables 1,2 and 3. It is clear that $\mu_{1}{ }^{\prime}, \mu_{2}$, and $\gamma$ are decreasing whereas C. V., $\sqrt{\beta_{1}}, \beta_{2}$ are increasing for increasing values of the parameter $\theta$.

Table 1. Numerical values of $\mu_{1}^{\prime}, \mu_{2}, \mathrm{C} . \mathrm{V}, \sqrt{\beta_{1}}, \beta_{2}$ and $\gamma$ of PLD, for varying values the parameter $\theta$.

\begin{tabular}{lllllll}
\hline \multicolumn{7}{l}{ Values of $\theta$ for Poisson-Lindley Distribution } \\
\hline \multicolumn{1}{l}{} & $\mathbf{2}$ & $\mathbf{3}$ & $\mathbf{4}$ & $\mathbf{5}$ & $\mathbf{6}$ \\
\hline$\mu_{1}{ }^{\prime}$ & 1.5 & 0.666667 & 0.416667 & 0.3 & 0.233333 & 0.190476 \\
$\mu_{2}$ & 3.25 & 1.055556 & 0.576389 & 0.385 & 0.285556 & 0.225624 \\
$\mathrm{CV}$ & 1.20185 & 1.541104 & 1.822087 & 2.068279 & 2.290174 & 2.493742 \\
$\sqrt{\beta_{1}}$ & 1.792108 & 2.083265 & 2.314307 & 2.517935 & 2.704839 & 2.87957 \\
$\beta_{2}$ & 7.532544 & 8.941828 & 10.10611 & 11.17187 & 12.19654 & 13.203 \\
$\gamma$ & 2.166667 & 1.583333 & 1.383333 & 1.283333 & 1.22381 & 1.184524 \\
\hline
\end{tabular}

Table 2. Numerical values of $\mu_{1}^{\prime}, \mu_{2}, \mathrm{C} . \mathrm{V}, \sqrt{\beta_{1}}, \beta_{2}$ and $\gamma$ of PSD for varying values the parameter $\theta$.

\begin{tabular}{lllllll}
\hline \multicolumn{7}{l}{ Values of $\theta$ for Poisson-Sujatha Distribution } \\
\hline & $\mathbf{1}$ & $\mathbf{2}$ & $\mathbf{3}$ & $\mathbf{4}$ & $\mathbf{5}$ & $\mathbf{6}$ \\
\hline$\mu_{1}{ }^{\prime}$ & 2.25 & 0.875 & 0.5 & 0.340909 & 0.25625 & 0.204545 \\
$\mu_{2}$ & 5.1875 & 1.484375 & 0.726190 & 0.451963 & 0.320586 & 0.246039 \\
$\mathrm{CV}$ & 1.012270 & 1.392399 & 1.704336 & 1.972026 & 2.209573 & 2.425006 \\
$\sqrt{\beta_{1}}$ & 1.467931 & 1.898599 & 2.205880 & 2.443548 & 2.646990 & 2.831109 \\
$\beta_{2}$ & 5.944113 & 7.864044 & 9.479441 & 10.777366 & 11.910905 & 12.969309 \\
$\gamma$ & 2.305555 & 1.696428 & 1.452381 & 1.325757 & 1.251067 & 1.202862 \\
\hline
\end{tabular}


Table 3. Numerical values of $\mu_{1}^{\prime}, \mu_{2}, \mathrm{C} . \mathrm{V}, \sqrt{\beta_{1}}, \beta_{2}$ and $\gamma$ of PAD for varying values the parameter $\theta$.

\begin{tabular}{lllllll}
\hline \multicolumn{7}{c}{ Values of $\boldsymbol{\theta}$ for Poisson-Amarendra Distribution } \\
\hline & $\mathbf{1}$ & $\mathbf{2}$ & $\mathbf{3}$ & $\mathbf{4}$ & $\mathbf{5}$ & $\mathbf{6}$ \\
\hline$\mu_{1}{ }^{\prime}$ & 3.3 & 1.181818 & 0.604167 & 0.382979 & 0.275904 & 0.214815 \\
$\mu_{2}$ & 7.61 & 2.14876 & 0.933594 & 0.528859 & 0.353998 & 0.262497 \\
$\mathrm{CV}$ & 0.835946 & 1.240347 & 1.599272 & 1.898871 & 2.156467 & 2.385051 \\
$\sqrt{\beta_{1}}$ & 1.172956 & 1.675185 & 2.106094 & 2.402408 & 2.626614 & 2.817693 \\
$\beta_{2}$ & 4.856217 & 6.666575 & 8.883016 & 10.59733 & 11.89457 & 12.99572 \\
$\gamma$ & 2.306061 & 1.818182 & 1.545259 & 1.38091 & 1.283048 & 1.221967 \\
\hline
\end{tabular}

The corresponding sub-figures of figure 2 depict the nature and behavior of the mean, the variance, coefficient of variation, coefficient of skewness, coefficient of kurtosis, and index of dispersion of the PLD, PSD and PAD for varying values of the parameter $\theta$. From these graphs it is obvious that the mean, variance and index of dispersion of PLD, PSD and PAD are decreasing whereas the corresponding coefficient of variation, coefficient of skewness, and coefficient of kurtosis are increasing for increasing values of the parameter $\theta$.
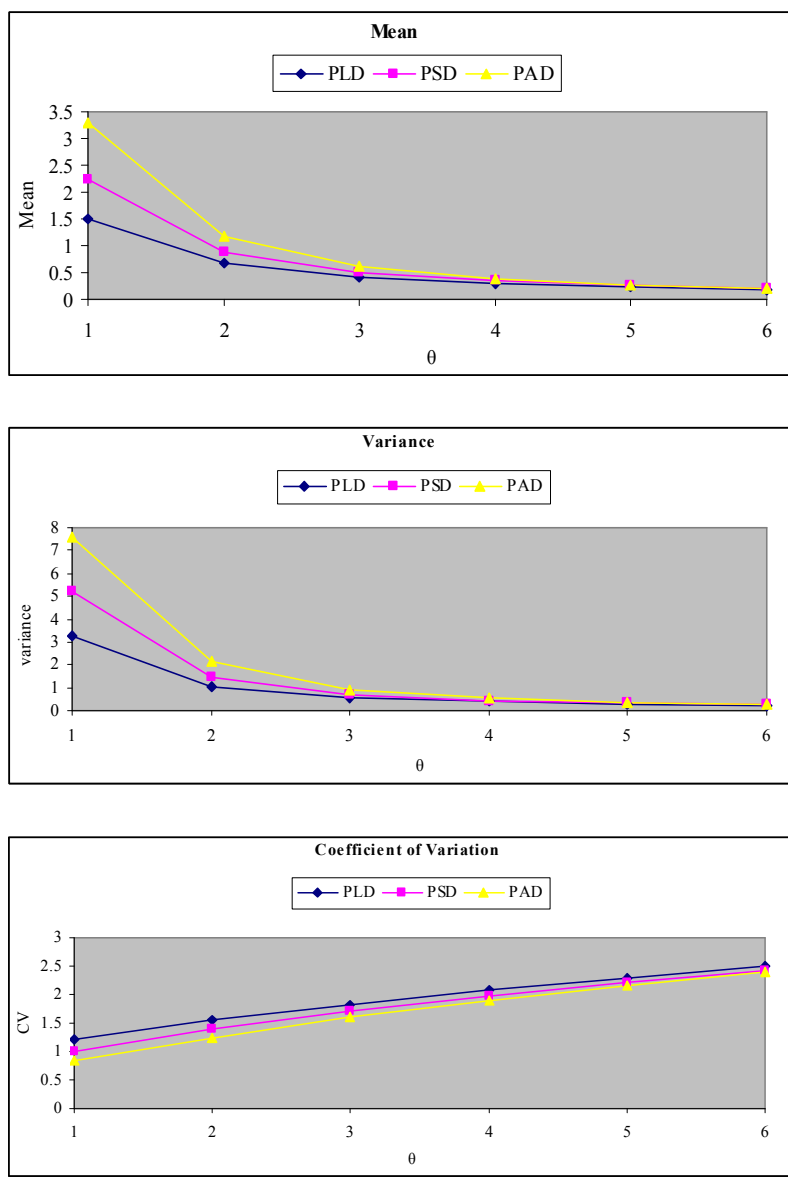
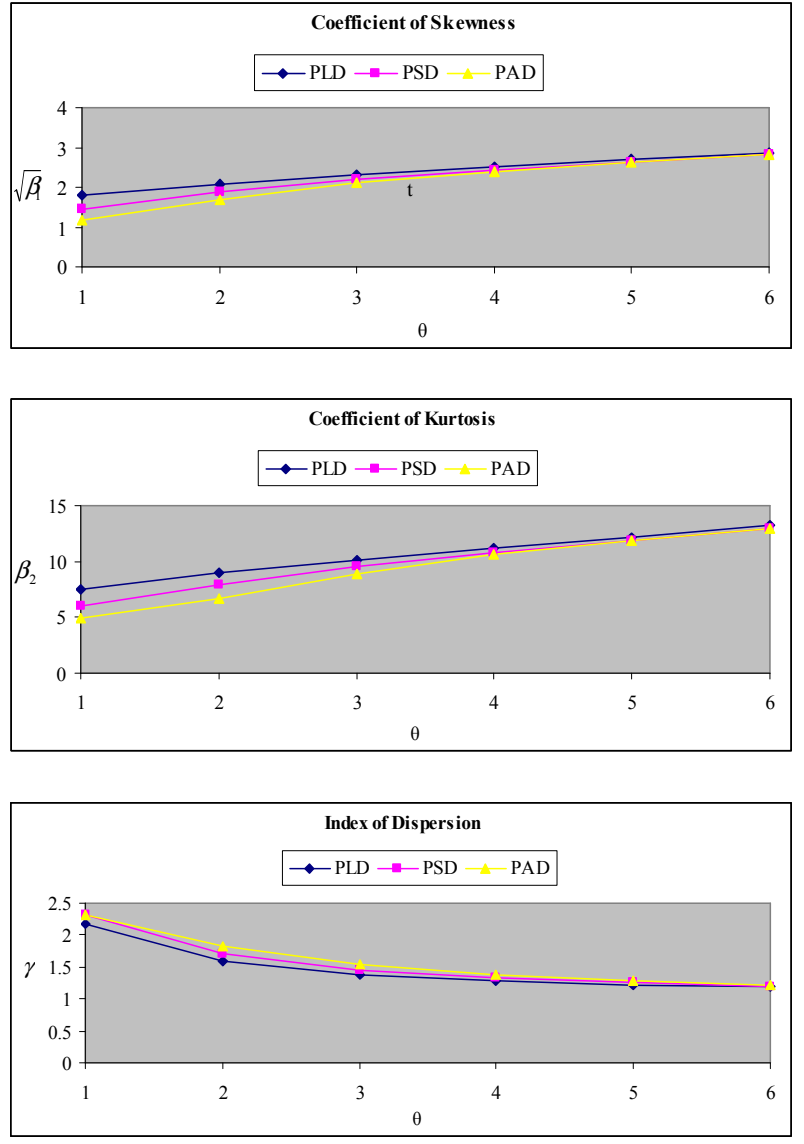

Fig. 2. Graphs of the mean, variance, coefficient of variation, coefficient of skewness, coefficient of kurtosis and Index of dispersion of the PLD, PSD and PAD for varying values of the parameter $\theta$.

\section{Statistical Properties}

\subsection{Increasing Hazard Rate (IHR) and Unimodality.}

The PAD as in (4) has an increasing hazard rate and has a unimodal density function. Since

$$
\frac{P(x+1 ; \theta)}{P(x ; \theta)}=\frac{1}{\theta+1}\left[1+\frac{3 x^{2}+(2 \theta+17) x+\left(\theta^{2}+6 \theta+16\right)}{x^{3}+(\theta+7) x^{2}+\left(\theta^{2}+5 \theta+15\right) x+\left(\theta^{3}+4 \theta^{2}+7 \theta+10\right)}\right]
$$

is decreasing function along $x, P(x ; \theta)$ is log-concave.

Therefore, the PAD has an increasing hazard rate (IHR) and its p. d. f. is unimodal. The interrelationship between logconcavity, unimodality and increasing hazard rate of discrete 
distributions has been discussed in Grandell (1997).

\subsection{Over-Dispersion}

The PAD (4) is always over- dispersed $\left(\sigma^{2}>\mu\right)$. We have

$$
\begin{gathered}
\sigma^{2}=\frac{\theta^{7}+4 \theta^{6}+14 \theta^{5}+58 \theta^{4}+144 \theta^{3}+156 \theta^{2}+240 \theta+144}{\theta^{2}\left(\theta^{3}+\theta^{2}+2 \theta+6\right)^{2}} \\
=\frac{\theta^{3}+2 \theta^{2}+6 \theta+24}{\theta\left(\theta^{3}+\theta^{2}+2 \theta+6\right)}\left[\frac{\theta^{7}+4 \theta^{6}+14 \theta^{5}+58 \theta^{4}+144 \theta^{3}+156 \theta^{2}+240 \theta+144}{\theta\left(\theta^{3}+\theta^{2}+2 \theta+6\right)\left(\theta^{3}+2 \theta^{2}+6 \theta+24\right)}\right] \\
=\mu\left[1+\frac{\theta^{6}+4 \theta^{5}+18 \theta^{4}+96 \theta^{3}+72 \theta^{2}+96 \theta+144}{\theta\left(\theta^{3}+\theta^{2}+2 \theta+6\right)\left(\theta^{3}+2 \theta^{2}+6 \theta+24\right)}\right]>\mu .
\end{gathered}
$$

This shows that PAD (4) is always over-dispersed.

\section{Parameter Estimation}

\subsection{Maximum Likelihood Estimate (MLE) of the Parameter}

Let $\left(x_{1}, x_{2}, \ldots, x_{n}\right)$ be a random sample of size $n$ from the corresponding to $X=x(x=\mathbf{1}, \mathbf{2}, \mathbf{3}, \ldots, k)$ such that $\sum_{x=1}^{k} f_{x}=n$, where $k$ is the largest observed value having nonzero frequency. The likelihood function $L$ of the PAD (4) is given by

PAD (4) and let $f_{x}$ be the observed frequency in the sample

$$
L=\left(\frac{\theta^{4}}{\theta^{3}+\theta^{2}+2 \theta+6}\right)^{n} \frac{1}{(\theta+1) \sum_{x=1}^{k} f_{x}(x+4)} \prod_{x=1}^{k}\left[\begin{array}{l}
x^{3}+(\theta+7) x^{2}+\left(\theta^{2}+5 \theta+15\right) x \\
+\left(\theta^{3}+4 \theta^{2}+7 \theta+10\right)
\end{array}\right]^{f_{x}}
$$

The log likelihood function is thus obtained as

$$
\log L=n \log \left(\frac{\theta^{4}}{\theta^{3}+\theta^{2}+2 \theta+6}\right)-\sum_{x=1}^{k} f_{x}(x+\mathbf{4}) \log (\theta+\mathbf{1})+\sum_{x=1}^{k} f_{x} \log \left[x^{3}+(\theta+7) x^{2}+\left(\theta^{2}+\mathbf{5} \theta+\mathbf{1 5}\right) x+\left(\theta^{3}+\mathbf{4} \theta^{2}+\mathbf{7} \theta+\mathbf{1 0}\right)\right]
$$

The first derivative of the log likelihood function is given by

$$
\frac{d \log L}{d \theta}=\frac{n\left(\theta^{3}+2 \theta^{2}+6 \theta+24\right)}{\theta\left(\theta^{3}+\theta^{2}+2 \theta+6\right)}-\frac{n(\bar{x}+4)}{\theta+1}+\sum_{x=1}^{k} \frac{\left[x^{2}+(2 \theta+5) x+\left(3 \theta^{2}+8 \theta+7\right)\right] f_{x}}{\left[x^{3}+(\theta+7) x^{2}+\left(\theta^{2}+5 \theta+15\right) x+\left(\theta^{3}+4 \theta^{2}+7 \theta+10\right)\right]}
$$

where $\bar{x}$ is the sample mean.

The maximum likelihood estimate (MLE), $\hat{\theta}$ of $\theta$ of PAD (4) is the solution of the equation $\frac{d \log L}{d \theta}=\mathbf{0}$ and is given by the solution of the following non-linear equation

$$
\begin{aligned}
& \frac{n\left(\theta^{3}+2 \theta^{2}+6 \theta+24\right)}{\theta\left(\theta^{3}+\theta^{2}+2 \theta+6\right)}-\frac{n(\bar{x}+4)}{\theta+1} \\
& +\sum_{x=1}^{k} \frac{\left[x^{2}+(2 \theta+5) x+\left(3 \theta^{2}+8 \theta+7\right)\right] f_{x}}{\left[x^{3}+(\theta+7) x^{2}+\left(\theta^{2}+5 \theta+15\right) x+\left(\theta^{3}+4 \theta^{2}+7 \theta+10\right)\right]}=\mathbf{0}
\end{aligned}
$$

This non-linear equation can be solved using any numerical iteration methods such as Newton - Raphson method, Bisection method, Regula - Falsi method etc. In this paper, Newton - Raphson method has been used to solve the above equation for estimating the parameter $\theta$.

\subsection{Method of Moment Estimate (MOME) of the Parameter}

Let $\left(x_{1}, x_{2}, \ldots, x_{n}\right)$ be a random sample of size $n$ from the PAD (4). Equating the population mean to the corresponding sample mean, the MOME $\tilde{\theta}$ of $\theta$ of PAD (4) is the solution of the following biquadratic equation

$$
\bar{x} \theta^{4}+(\bar{x}-1) \theta^{3}+2(\bar{x}-1) \theta^{2}+6(\bar{x}-1) \theta-24=0
$$

where $\bar{x}_{\text {is the sample mean. }}$

\section{Applications and Goodness of Fit}

In this section the PAD is fitted through four count data 
sets in order to test the PAD's goodness of fit in applications and the fiting are then compared with that of Poisson distribution (PD), Poisson-Lindley distribution (PLD) and Poisson-Sujatha distribution (PSD). The corresponding maximum likelihood estimates (MLE) were used to fit those distributions. The first data set in table 4 is due to Beall (1940) regarding the distribution of Pyrausta nublilalis, while the data sets in table 5,6 and 7 are relating to the observed number of days that experienced $\mathrm{X}$ thunderstorms events at Cape Kennedy, Florida for the 11 year period of record for the months of June, July and August, January 1957 to December, 1967, available in Falls et al (1971). From the fitting of these distributions, it is obvious that PAD provides quite satisfactory fit in all data sets and hence PAD can be considered as an important distribution for modeling data sets over PD, PLD, and PSD.

Table4. Distribution of Pyrausta nubilalis in 1937.

\begin{tabular}{|c|c|c|c|c|c|}
\hline \multirow{2}{*}{ No. of insects } & \multirow{2}{*}{ Observed Frequency } & \multicolumn{4}{|c|}{ Expected Frequency } \\
\hline & & PD & PLD & PSD & PAD \\
\hline & & 26.4 & 31.5 & 31.5 & 31.6 \\
\hline 0 & 33 & 19.8 & 14.2 & 14.2 & 14.0 \\
\hline 1 & 12 & 7.4 & 6.1 & 6.1 & 6.1 \\
\hline 2 & 6 & 18 & 2.5 & 2.6 & 2.8 \\
\hline $\begin{array}{l}3 \\
4\end{array}$ & $\begin{array}{l}3 \\
1\end{array}$ & $\left.\begin{array}{l}1.0 \\
0.3\end{array}\right\}$ & $\begin{array}{l}2.3 \\
1.0\end{array}$ & $\left.\begin{array}{l}2.0 \\
1.0\end{array}\right\}$ & 1.0 \\
\hline 5 & 1 & $\begin{array}{l}0.0 \\
0.3\end{array}$ & $\begin{array}{l}0.0 \\
0.7\end{array}$ & & 1.5 \\
\hline Total & 56 & 56.0 & 56.0 & 56.0 & 56.0 \\
\hline ML estimate & & $\hat{\theta}=\mathbf{0 . 7 5 0 0}$ & $\hat{\theta}=1.8081$ & $\hat{\theta}=\mathbf{2 . 2 4 1 4 5 4}$ & $\hat{\theta}=2.640279$ \\
\hline$\chi^{2}$ & & 4.87 & 0.53 & 0.45 & 0.36 \\
\hline d. f. & & 1 & 1 & 1 & 2 \\
\hline p-value & & 0.0273 & 0.4666 & 0.5023 & 0.8353 \\
\hline
\end{tabular}

Table 5. Observed and expected number of days that experienced X thunderstorms events at Cape Kennedy, Florida for the 11-year period of record for the month of June, January 1957 to December 1967, Falls et al (1971).

\begin{tabular}{|c|c|c|c|c|c|}
\hline \multirow{2}{*}{ No. of Thunderstorms } & \multirow{2}{*}{$\begin{array}{l}\text { Observed } \\
\text { Frequency }\end{array}$} & \multicolumn{4}{|c|}{ Expected Frequency } \\
\hline & & PD & PLD & PSD & PAD \\
\hline & & 155.6 & 185.3 & 184.8 & 185.4 \\
\hline 0 & 187 & 117.0 & 83.5 & 83.6 & 82.7 \\
\hline 1 & 77 & 43.9 & 35.9 & 36.3 & 36.3 \\
\hline 2 & 40 & 11.0 & 15.0 & 15.2 & 15.4 \\
\hline $\begin{array}{l}3 \\
4\end{array}$ & $\begin{array}{l}17 \\
6\end{array}$ & 2.1 & 6.1 & $6.1)$ & 6.3 \\
\hline 5 & 2 & 0.3 & $2.5\}$ & $2.4\}$ & $2.4\}$ \\
\hline 6 & 1 & 0.1 & 1.7 & 1.6 & 1.5 \\
\hline Total & 330 & 330.0 & 330.0 & 330.0 & 330.0 \\
\hline ML estimate & & $\hat{\theta}=0.751515$ & $\hat{\theta}=1.804268$ & $\hat{\theta}=2.229891$ & $\hat{\theta}=2.625345$ \\
\hline$\chi^{2}$ & & 31.93 & 1.43 & 1.25 & 1.07 \\
\hline d. f. & & 2 & 3 & 3 & 3 \\
\hline $\mathrm{p}$-value & & 0.0000 & 0.6985 & 0.7410 & 0.7843 \\
\hline
\end{tabular}

Table 6. Observed and expected number of days that experienced X thunderstorms events at Cape Kennedy, Florida for the 11-year period of record for the month of July, January 1957 to December 1967, Falls et al (1971).

\begin{tabular}{|c|c|c|c|c|c|}
\hline \multirow{2}{*}{ No. of Thunderstorms } & \multirow{2}{*}{$\begin{array}{l}\text { Observed } \\
\text { Frequency }\end{array}$} & \multicolumn{4}{|c|}{ Expected Frequency } \\
\hline & & PD & PLD & PSD & PAD \\
\hline 0 & 177 & 142.3 & 177.7 & 176.5 & 176.7 \\
\hline 0 & $\begin{array}{l}177 \\
80\end{array}$ & 124.4 & 88.0 & 88.4 & 87.6 \\
\hline $\begin{array}{l}1 \\
2\end{array}$ & $\begin{array}{l}80 \\
47\end{array}$ & 54.3 & 41.5 & 42.2 & 42.3 \\
\hline $\begin{array}{l}2 \\
3\end{array}$ & $\begin{array}{l}4 / \\
26\end{array}$ & 15.8 & 18.9 & 19.2 & 19.5 \\
\hline 4 & 9 & 3.5 & $8.4\}$ & 8.5 & 8.6 \\
\hline 5 & 2 & 0.7 & $6.5\}$ & $6.2\}$ & $6.3\}$ \\
\hline Total & 341 & 341.0 & 341.0 & 341.0 & 341.0 \\
\hline ML estimate & & $\hat{\theta}=\mathbf{0 . 8 7 3 9 0 0}$ & $\hat{\theta}=\mathbf{1 . 5 8 3 5 3 6}$ & $\hat{\theta}=1.995806$ & $\hat{\theta}=\mathbf{2 . 3 9 0 4 7 4}$ \\
\hline$\chi^{2}$ & & 39.74 & 5.15 & 4.67 & 4.35 \\
\hline d. f. & & 2 & 3 & 3 & 3 \\
\hline p-value & & 0.0000 & 0.1611 & 0.1976 & 0.2261 \\
\hline
\end{tabular}


Table 7. Observed and expected number of days that experienced $X$ thunderstorms events at Cape Kennedy, Florida for the 11-year period of record for the month of August, January 1957 to December 1967, Falls et al (1971).

\begin{tabular}{|c|c|c|c|c|c|}
\hline \multirow{2}{*}{ No. of Thunderstorms } & \multirow{2}{*}{$\begin{array}{l}\text { Observed } \\
\text { Frequency }\end{array}$} & \multicolumn{4}{|c|}{ Expected Frequency } \\
\hline & & PD & PLD & PSD & PAD \\
\hline & & 151.8 & 184.8 & 184.1 & 184.7 \\
\hline 0 & $\begin{array}{l}185 \\
89\end{array}$ & 122.9 & 87.2 & 87.5 & 86.6 \\
\hline $\begin{array}{l}1 \\
2\end{array}$ & $\begin{array}{l}89 \\
30\end{array}$ & 49.7 & 39.3 & 39.8 & 39.8 \\
\hline 3 & $\begin{array}{l}30 \\
24\end{array}$ & 13.4 & 17.1 & 17.3 & 17.6 \\
\hline 4 & 10 & 2.7 & $7.3\}$ & 7.3 & $7.4\}$ \\
\hline 5 & 3 & 0.5 & $5.3\}$ & $5.0\}$ & $4.9\}$ \\
\hline Total & 341 & 341.0 & 341.0 & 341.0 & 341.0 \\
\hline ML estimate & & $\hat{\theta}=\mathbf{0 . 8 0 9 3 8 4}$ & $\hat{\theta}=1.693425$ & $\hat{\theta}=\mathbf{2 . 1 1 4 5 4 5}$ & $\hat{\theta}=2.511962$ \\
\hline$\chi^{2}$ & & 49.49 & 5.03 & 5.06 & 4.83 \\
\hline d. f. & & 2 & 3 & 3 & 3 \\
\hline p-value & & 0.0000 & 0.1696 & 0.1674 & 0.1847 \\
\hline
\end{tabular}

\section{Concluding Remarks}

In the present paper the Poisson-Amarendra distribution (PAD) has been proposed by compounding Poisson distribution with Amarendra distribution, introduced by Shanker (2016 c). The $r$ th factorial raw moment has been derived and the first four raw moments and the central moments have been given. The expression of the coefficient of variation, skewness, kurtosis and index of dispersion has also been obtained. The estimation of its parameter has been discussed using both maximum likelihood estimation and the method of moments. The PAD has been fitted using maximum likelihood estimate to certain data sets to test its goodness of fit over Poisson distribution (PD), PoissonLindley distribution (PLD) and Poisson-Sujatha distribution (PSD). It is found that Poisson-Amarendra distribution (PAD) provides better fit than PD, PLD, and PSD for all data sets.

\section{Acknowledgements}

The author expresses his thankfulness to the anonymous reviewers for their constructive comments which improved the quality of the paper

\section{References}

[1] Beall, G. (1940): The fit and significance of contagious distributions when applied to observations on larval insects, Ecology, 21, 460-474.

[2] Falls, L. W., Williford, W. O. and Carter, M. C. (1971): Probability distributions for thunderstorm activity at Cape Kennedy, Florida, Journal of Applied Meteorology, 10, 97104.

[3] Grandell, J. (1997): Mixed Poisson Processes, Chapman \& Hall, London.
[4] Lindley, D. V. (1958): Fiducial distributions and Bayes theorem, Journal of the Royal Statistical Society, 20 (1), 102107.

[5] Sankaran, M. (1970): The discrete Poisson-Lindley distribution, Biometrics, 26, 145-149.

[6] Shanker, R. (2015 a): Akash distribution and Its Applications, International Journal of Probability and Statistics, 4 (3), 6575 .

[7] Shanker, R. (2015 b): Shanker distribution and Its Applications, International Journal of Statistics and Applications, 5 (6), 338-348.

[8] Shanker, R. (2016 a): Sujatha distribution and Its Applications, to appear in "Statistics in Transition new Series", 17 (3).

[9] Shanker, R. (2016 b): The Discrete Poisson-Sujatha distribution, International Journal of Probability and Statistics, 5 (1), 1-9.

[10] Shanker, R. (2016 c): Amarendra distribution and Its Applications, American Journal of Mathematics and Statistics, 6 (1), 44-56.

[11] Shanker, R. and Hagos, F. (2015): On Poisson-Lindley distribution and its Applications to Biological Sciences, Biometrics and Biostatistics International Journal, 2 (4), 1-5.

[12] Shanker, R. and Hagos, F. (2016 a): On Poisson-Sujatha distribution and Its Applications to model count data from Biological Sciences, Biometrics \& Biostatistics International Journal, 3 (4), 1-7.

[13] Shanker, R. and Hagos, F. (2016 b): Size-biased PoissonSujatha distribution with Applications, American Journal of Mathematics and Statistics 6 (4), 145-154.

[14] Shanker, R. and Hagos, F (2016 c): Zero-truncated PoissonSujatha distribution with Applications, Communicated

[15] Shanker, R. and Hagos, F. (2016 d): On zero-truncation of Poisson, Poisson-Lindley, and Poisson-Sujatha distributions and Their Applications, Biometrics \& Biostatistics International Journal, 3 (5), 1-13. 